\title{
AVALIAÇÃO DAS PROPRIEDADES DE COMPÓSITOS DE POLIPROPILENO REFORÇADOS COM CASCA DE AVEIA
}

\author{
Luciano Gomes Furlan \\ Instituto Federal do Rio Grande do Sul, Campus Restinga, Estrada João Antônio da Silveira, 351, 91790-400 Porto Alegre - RS, Brasil \\ Ueiler Lisoski Duarte e Raquel Santos Mauler* \\ Departamento de Química orgânica, Instituto de Química, Universidade Federal do Rio Grande do Sul, Av. Bento Gonçalves, \\ 9500, 91501-970 Porto Alegre - RS, Brasil
}

Recebido em 27/7/11; aceito em 5/4/12; publicado na web em 2/7/12

\begin{abstract}
EVALUATION OF OAT-REINFORCED POLYPROPYLENE COMPOSITE PROPERTIES. The preparation of oat-reinforced polypropylene nanocomposites with different fiber contents by means of melt-processing was investigated. Composite properties were evaluated by Scanning Electron Microscopy (SEM), Flexural Modulus, Dynamic Mechanical Analysis (DMA), Differential Scanning Calorimetry (DSC) and Thermogravimetric Analysis (TGA). Findings confirmed that the oat composite properties were affected by fiber type and content. Improvements in mechanical properties were obtained using fiber contents $\leq 20 \%$ w.t.
\end{abstract}

Keywords: polypropylene; oat; mechanical properties.

\section{INTRODUÇÃO}

Nos últimos anos, nossa sociedade passou a preocupar-se em desenvolver tecnologias mais "verdes", com o intuito de reduzir impactos ao meio ambiente e suas consequências. O Brasil possui um grande potencial na produção de recursos renováveis, como produtos agrícolas, florestais e resíduos lignocelulósicos (são exemplos o bagaço de cana-de-açúcar, as palhas de trigo e arroz, a casca de aveia e as aparas de madeira). Neste sentido, o emprego destes materiais como reforço de polímeros tem crescido substancialmente, ${ }^{1}$ devido a alguns fatores importantes como baixo custo, baixa densidade, boa resistência térmica e mecânica, serem biodegradáveis e, principalmente, por serem provenientes de fontes renováveis. ${ }^{2,3}$ Por esses fatores tornaram-se uma alternativa interessante, podendo substituir reforços inorgânicos obtidos de fontes não renováveis, como as fibras sintéticas (vidro, aramida, carbono etc). Além disso, apresentam vantagens adicionais por serem mais leves e menos abrasivas aos equipamentos de processamento. São exemplos os compósitos de farelo de madeira, ${ }^{4}$ cana, juta, ${ }^{5}$ coco, ${ }^{6}$ bananeira ${ }^{7}$ e sisal, ${ }^{8}$ entre outros. O Brasil destaca-se mundialmente pela produção de compósitos utilizando fibras vegetais, ${ }^{9}$ principalmente destinadas aos setores da construção civil e automobilística. Por exemplo, compósitos contendo fibras naturais são utilizados em muitas partes dos revestimentos internos de veículos ou incorporados a produtos para redução de custos finais. Uma limitação da incorporação de fibras naturais em materiais poliméricos são as perdas na sua resistência mecânica, muitas vezes em virtude da fraca interação entre o polímero e a fibra $\mathrm{O}$ uso de tratamentos químicos ${ }^{10}$ nas fibras melhora significativamente a adesão interfacial fibra/matriz. ${ }^{11}$ Por exemplo, o tratamento alcalino remove impurezas naturais e artificiais produz rugosidade na superfície da fibra, ${ }^{12}$ aumenta a razão de aspecto causada pela redução do diâmetro da fibra, ${ }^{13}$ podendo aumentar em até $30 \%$ propriedades mecânicas como tração e módulo. ${ }^{14}$

Nos dias de hoje, a produção mundial anual de aveia é bastante expressiva, em torno de 50 milhões de toneladas por ano. No entanto, o processamento de aveia para consumo humano gera resíduos, cascas, pelos que se desprendem dos grãos e parte do endosperma. A produção desse resíduo pode atingir $50 \%$ do peso da planta ${ }^{15} \mathrm{e}$

*e-mail: mauler@iq.ufrgs.br não possui valor econômico. Uma alternativa é sua aplicação para a alimentação animal, mas ainda é insuficiente para consumir todos os resíduos gerados. A casca da aveia tem sido descartada durante o processamento do grão e torna-se, com isso, um poluente ao meio ambiente. ${ }^{16}$ Diante do problema apresentado, este trabalho teve como objetivos a produção de compósitos de polipropileno reforçados com diferentes teores de casca de aveia e a avaliação de suas propriedades mecânicas, concedendo aos resíduos de casca de aveia outros destinos diferentes do lixo comum.

\section{PARTE EXPERIMENTAL}

\section{Materiais}

Utilizou-se polipropileno comercial, fornecido pela Braskem S.A., com índice de fluidez $3,5 \mathrm{~g} 10^{-1} \mathrm{~min}^{-1}\left(230{ }^{\circ} \mathrm{C}\right.$ e $\left.2.16 \mathrm{~kg}\right)$ e peso molecular de $420 \mathrm{~kg} \mathrm{~mol}^{-1}$. A casca de aveia foi gentilmente fornecida pela Quaker S.A. Para a produção dos compósitos foram usados dois tipos de casca de aveia: na forma bruta, como recebida, e com classificação granulométrica obtida através de peneiramento (mesh 35) da casca de aveia. As amostras de aveia foram tratadas em solução de hidróxido de sódio 5\% por $1 \mathrm{~h}$, à temperatura ambiente, sob agitação mecânica. Após, foram lavadas em água destilada várias vezes e secas em estufa a vácuo a $50{ }^{\circ} \mathrm{C}$ por $24 \mathrm{~h}$.

\section{Preparação dos compósitos e caracterização}

Os compósitos foram preparados em câmara de mistura fechada (Haake, modelo Rheomix 600p) equipada com reômetro de torque e dois rotores contrarrotatórios operando a 50 rpm e temperatura de 180 ${ }^{\circ} \mathrm{C}$. Uma mistura (com massa total de $40 \mathrm{~g}$ ) contendo o polipropileno e diferentes porcentagens das amostras de casca de aveia (bruta e com classificação granulométrica de mesh 35) foi processada durante 7 min. A massa total presente na câmara de mistura foi de $40 \mathrm{~g}$.

Os corpos de prova (em formato gravata) para a realização dos ensaios mecânicos foram preparados por compressão, utilizando-se uma prensa hidráulica Carver modelo 3710C, onde os compósitos foram prensados durante $3 \mathrm{~min}$, a uma pressão de $2.500 \mathrm{lbf}$ e temperatura de $190{ }^{\circ} \mathrm{C}$. 
As micrografias dos compósitos, fraturados em nitrogênio líquido, foram obtidas por microscopia eletrônica de varredura (MEV) em um equipamento JEOL modelo JSM 6060, operando a uma voltagem de $10 \mathrm{kV}$.

Os ensaios de tração foram realizados numa máquina universal de ensaios EMIC modelo DL 10000, com velocidade de $5 \mathrm{~mm} / \mathrm{min}$ e célula de carga de $5 \mathrm{kN}$, de acordo com a ASTM D 63890.

O módulo de flexão e a temperatura de transição vítrea $(\mathrm{Tg})$ dos compósitos foram determinados por ensaios de análise dinâmico-mecânica (DMA) em um equipamento TA Instruments modelo Q800, em modo single cantilever de -30 a $130^{\circ} \mathrm{C}$, com taxa de aquecimento de $3{ }^{\circ} \mathrm{C} \mathrm{min}{ }^{-1}$ a uma frequência fixa de $1 \mathrm{~Hz}$.

A temperatura de fusão cristalina (Tm), o grau de cristalinidade $\left(\chi_{\mathrm{c}}\right)$ e a temperatura de cristalização (Tc) foram determinados por calorimetria diferencial de varredura (DSC) no equipamento 2100 Thermal Analyst Instruments, Perkin Elmer. As amostras foram aquecidas de 50 até $200{ }^{\circ} \mathrm{C}$, com taxa de aquecimento/resfriamento de $10{ }^{\circ} \mathrm{C} \mathrm{min}^{-1}$ e sob atmosfera de nitrogênio.

\section{RESULTADOS E DISCUSSÃO}

\section{Morfologia}

As imagens das superfícies fraturadas por criogenia (Figuras $1 \mathrm{e}$ 2), obtidas por microscopia eletrônica de varredura (MEV), mostraram a existência de lacunas (buracos) entre as fibras e a matriz de PP provenientes do descolamento das mesmas, devido à baixa adesão interfacial e pouca molhabilidade da fibra no interior da matriz. Isto ocorre, provavelmente, devido à grande diferença de energia superficial entre as duas fases. Este efeito é mais pronunciado nos compósitos sem classificação granulométrica (aveia bruta), em virtude da presença de partículas de diferentes tamanhos. Os compósitos produzidos com casca de aveia peneirada com classificação granulométrica (mesh 35) apresentaram maior homogeneidade.

Como esperado, observou-se baixa adesão entre as fibras e a matriz de PP sem o uso de agentes compatibilizantes, devido à natureza hidrofílica das cascas de aveia e hidrofóbica do PP. Percebe-se que a aglomeração das fibras promoveu falhas nos compósitos, além da presença de fibras descoladas.

As propriedades dos compósitos de polipropileno contendo diversos teores de casca de aveia são apresentadas na Tabela 1. Comparada ao PP virgem, a adição de casca de aveia não provocou alterações na temperatura de fusão cristalina (Tm) dos compósitos. Por outro lado, verificou-se o efeito nucleante que a fibra exerce sobre a matriz polimérica, comprovado pelo aumento da temperatura de cristalização (Tc) dos compósitos. A nucleação geralmente diminui o tamanho

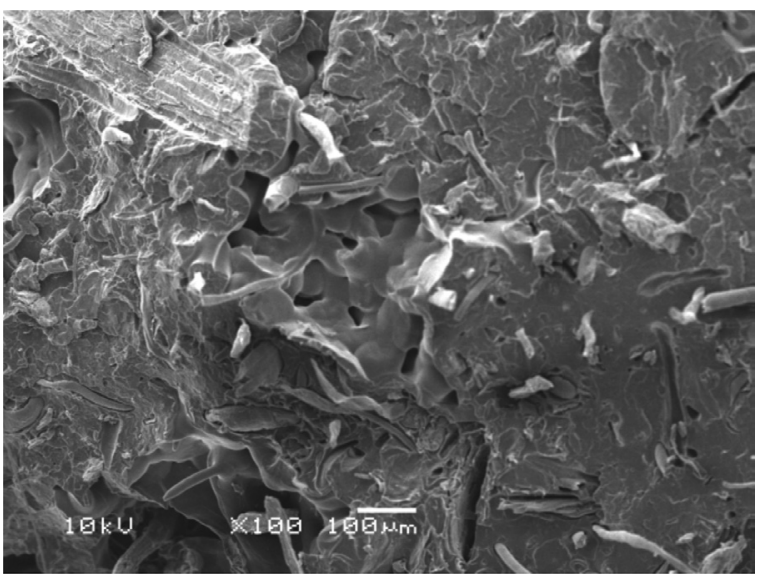

Figura 1. Fotomicrografias MEV de superfícies criofraturadas dos compósitos de aveia (mesh 35 ) contendo $20 \%$ de aveia

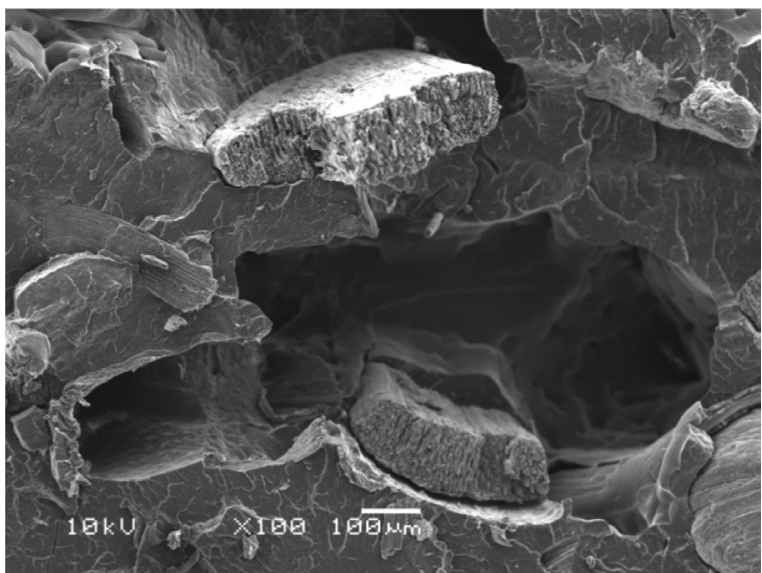

Figura 2. Fotomicrografias MEV de superfícies criofraturadas dos compósitos de aveia (bruta) contendo $20 \%$ de aveia

dos esferulitos, acarretando no aumento da Tc dos materiais. Devido à maior área superficial da casca de aveia peneirada (mesh 35), o aumento na Tc foi levemente superior nos compósitos com teores $\geq 20 \%$, independente da quantidade de casca de aveia.

O grau de cristalinidade $\left(\chi_{\mathrm{c}}\right)$ decresce com o aumento da quantidade de fibras nas amostras. Teores mais elevados de carga implicam na formação de cristais menores e menos perfeitos, devido a um maior número de impedimentos ao seu crescimento, resultando no decréscimo da $\chi_{c}$.

Não se observou variação significativa na temperatura de transição

Tabela 1. Propriedades dos compósitos de PP contendo diferentes quantidades de casca de aveia

\begin{tabular}{|c|c|c|c|c|c|c|c|}
\hline $\begin{array}{c}\% \text { de casca } \\
\text { de aveia }\end{array}$ & $\begin{array}{l}\mathrm{Tm} \\
\left({ }^{\circ} \mathrm{C}\right)\end{array}$ & $\begin{array}{c}\mathrm{c} \\
\left({ }^{\circ} \mathrm{C}\right)\end{array}$ & $\begin{array}{l}\chi_{\mathrm{c}} \\
(\%)\end{array}$ & $\begin{array}{l}\mathrm{Tg} \\
\left({ }^{\circ} \mathrm{C}\right)\end{array}$ & $\begin{array}{l}\text { Temperatura perda } \\
\text { de } 10 \% \text { massa }\left({ }^{\circ} \mathrm{C}\right)\end{array}$ & $\begin{array}{c}\text { Módulo de } \\
\text { armazenamento }(\mathrm{MPa})\end{array}$ & $\begin{array}{c}\text { Módulo elástico } \\
(\mathrm{MPa})\end{array}$ \\
\hline 0 & 164 & 111 & 52 & 11 & 365 & 1342 & $1207 \pm 58$ \\
\hline$* 10$ & 165 & 114 & 44 & 11 & 354 & 1809 & $1075 \pm 40$ \\
\hline$* 20$ & 165 & 115 & 38 & 11 & 323 & 1787 & $1391 \pm 68$ \\
\hline$* 30$ & 165 & 116 & 34 & 9 & 297 & 651 & $1151 \pm 33$ \\
\hline$* 40$ & 165 & 115 & 25 & 9 & 289 & 763 & $1083 \pm 81$ \\
\hline$* \mathbf{5 0}$ & 166 & 115 & 18 & 11 & 292 & 678 & $1051 \pm 45$ \\
\hline 10 & 169 & 111 & 35 & 12 & 376 & 1512 & $945 \pm 77$ \\
\hline 20 & 167 & 113 & 34 & 11 & 324 & 1611 & $1049 \pm 70$ \\
\hline 30 & 168 & 113 & 33 & 9 & 317 & 485 & $1123 \pm 58$ \\
\hline 40 & 167 & 114 & 25 & 12 & 297 & 558 & $1188 \pm 90$ \\
\hline 50 & 167 & 114 & 22 & 12 & 306 & 613 & $1059 \pm 95$ \\
\hline
\end{tabular}

(*) Compósitos produzidos com casca de aveia peneirada com classificação granulométrica - mesh 35. 
vítrea (Tg) dos compósitos. Por outro lado, a presença da casca de aveia reduziu a estabilidade térmica do material, comprovada pela determinação da temperatura de perda de $10 \%$ de massa dos compósitos. Este efeito foi mais pronunciado em teores de aveia $\geq 30 \%$. A perda de estabilidade térmica pode estar associada à ocorrência de degradação das fibras e formação de espaços vazios devido à presença de substâncias voláteis. ${ }^{17,18}$

Os resultados dos ensaios de tração mostraram que a adição das fibras ao PP resulta em uma diminuição da resistência do material, pois os compósitos suportam uma tensão máxima inferior ao PP puro (Figura 3). Este fator é provocado por descontinuidades na matriz polimérica, devido ao aumento da quantidade de fibras presentes. Também se deve levar em consideração a fraca interação dos constituintes. A baixa resistência verificada nos compósitos não compatibilizados também é resultante de falhas na sua interface, ocasionadas pela fraca interação dos constituintes.

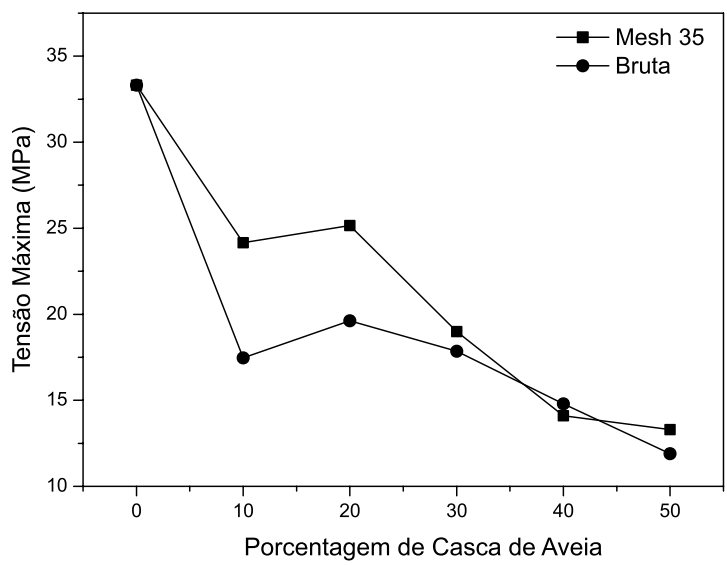

Figura 3. Tensão máxima $(\mathrm{MPa})$ dos compósitos produzidos com diferentes porcentagens de casca de aveia

O módulo elástico dos compósitos não é influenciado significativamente por tipo e quantidade de casca de aveia. Por outro lado, o efeito do reforço é mais pronunciado no módulo de armazenamento (Figura 4), onde existe um aumento do módulo nos compósitos com teores de até $20 \%$. Observou-se um incremento máximo de $35 \%$ no módulo de armazenamento (compósito contendo $10 \%$ de casca de aveia peneirada com mesh 35) indicando que a fibra atua como carga de reforço, aumentando a rigidez do material. No entanto, verificou-se perda de resistência mecânica para teores de fibra superiores a $30 \%$.

Utilizando teores de aveia de até $20 \%$, os compósitos de PP reforçados com casca de aveia apresentaram um bom balanço das

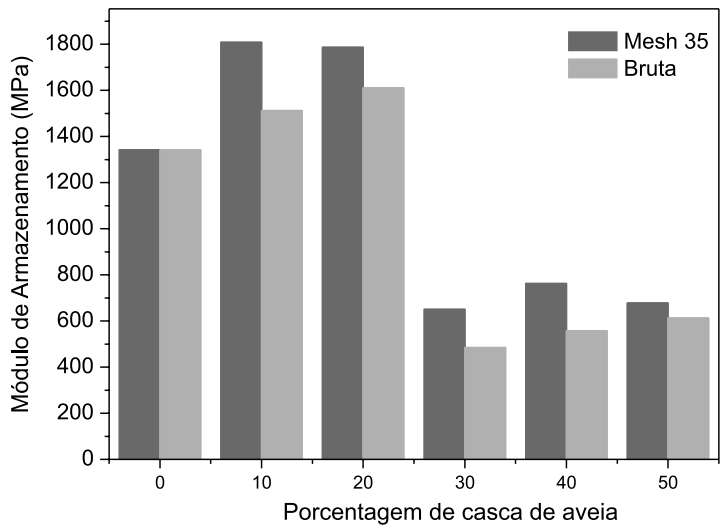

Figura 4. Módulo de armazenamento dos compósitos produzidos com diferentes porcentagens de casca de aveia propriedades mecânicas, pois a matriz transfere para a fibra a tensão aplicada de forma homogênea, aumentando com isso a rigidez do material. No entanto, em porcentagens de aveia superiores a $20 \%$, a aglomeração das fibras na matriz durante o processamento leva à perda de resistência mecânica do material, ocasionando a formação de falhas na peça injetada.

\section{CONCLUSÕES}

Este trabalho mostrou uma alternativa para o reaproveitamento de resíduos do processamento industrial da aveia, através da produção de compósitos de polipropileno. As amostras obtidas com cascas de aveia peneirada (mesh 35) foram aquelas que apresentaram melhor adesão e incorporação da fibra na matriz polimérica, proporcionando boa resistência mecânica com até $20 \%$ de fibra. No entanto, porcentagens superiores a $30 \%$ de casca de aveia reduzem a resistência mecânica do material

\section{AGRADECIMENTOS}

À Quaker S.A. pelo fornecimento da aveia, Braskem S.A, CNPq, FAPERGS e FINEP pelo financiamento e suporte técnico ao projeto.

\section{REFERÊNCIAS}

1. Jawaid, M.; Abdul Khalil, H. P. S.; Carbohydr. Polym. 2011, 86, 1.

2. Ku, H.; Wang, H.; Pattarachaiyakoop, N.; Trada, M.; Composites, Part B 2011, 42, 856 .

3. Satyanarayana, K. G.; Guimarães, J. L.; Wypych, F.; Compos. Part A 2007, 38, 1694.

4. Correa, C. A.; Fonseca, C. N. P.; Neves, S.; Razzino, C. A.; Hage, E.; Polim.: Cienc. Tecnol. 2003, 13, 154.

5. Hong, C. K.; Hwang, I.; Kim, N.; Park, D. H.; Hwang, B. S.; Nah, C.; J. Ind. Eng. Chem. 2008, 14, 71.

6. Santos, E. F.; Moresco, M.; Rosa, S. M. L.; Nachtigall, S. M. B.; Polímeros 2010, 20, 215; Rozman, H. D.; Tan, K. W.; Kumar, R. N.; Abubakar, A.; Ishak, Z.; Ismail, H.; Eur. Polym. J. 2000, 36, 1483; Wambua, P.; Ivens, J.; Verpoest, I.; Compos. Sci. Technol. 2003, 63,1259; Ishizaki, M. H.; Visconte, L. L. Y.; Furtado, C. R. G.; Leite, M. C.; Leblanc, J. L.; Polim.: Cienc. Tecnol. 2006, 16, 182.

7. Becker, D.; Kleinschmidt, A. C.; Balzer, P. S.; Polímeros 2011, $21,7$.

8. Spinacé, M. A. S.; Janeiro, L. D.; Bernardino, F. C.; Grossi, T. A.; De Paoli, M.; Polímeros 2011, 21, 168.

9. Silva, R.; Haraguchi, S. H.; Muniz, E.C.; Rubira, A.; Quim. Nova 2009, 32, 661.

10. Zheng, Y.; Cao, D.; Wang, D.; Chen, A.; Compos. Part A 2007, 38, 20; Eichhron, S. J.; Baillie, C. A.; Zafeiropoulos, N.; Mwaikambo, L. Y.; Ansell, M. P.; Dufresne, A.; Entwistle, K. M.; Herrera-franco, P. J.; J. Mater. Sci. 2001, 36, 2107.

11. Bledzki, A.K.; Gassan, J.; Prog. Polym. Sci. 1999, 24, 221.

12. Mohanty, A. K.; Wibowo, A.; Misra, M.; Drzal, L. T.; Compos. Part A 2004, 35, 363

13. Li, Y.; Mai, Y.- W.; Ye, L.. Compos. Sci. Tech. 2000, 60, 2037.

14. Ray, D.; Sarkar, B. K.; Das, S.; Rana, A. K.; Compos. Sci. Technol. 2002, 63, 911.

15. http://pt.calameo.com/read/000200968cc3a949579a0, acessada em Junho 2012

16. Morris, C. F.; Stephen, M. A.; Cereal Chem. 1997, 74, 379.

17. Santos, E. F.; Dissertação de Mestrado, Universidade Federal do Rio Grande do Sul, Brasil, 2007.

18. Bonelli, C. C.; Elzubair, A.; Suarez, J. C. M.; Mano, E. B.; Polim.: Cienc. Tecnol. 2005, 15, 256. 


\section{AVALIAÇÃO DAS PROPRIEDADES DE COMPÓSITOS DE POLIPROPILENO REFORÇADOS COM CASCA DE AVEIA}

\section{Luciano Gomes Furlan}

Instituto Federal do Rio Grande do Sul, Campus Restinga, Estrada João Antônio da Silveira, 351, 91790-400 Porto Alegre - RS, Brasil Ueiler Lisoski Duarte e Raquel Santos Mauler*

Departamento de Química orgânica, Instituto de Química, Universidade Federal do Rio Grande do Sul, Av. Bento Gonçalves, 9500, 91501-970 Porto Alegre - RS, Brasil

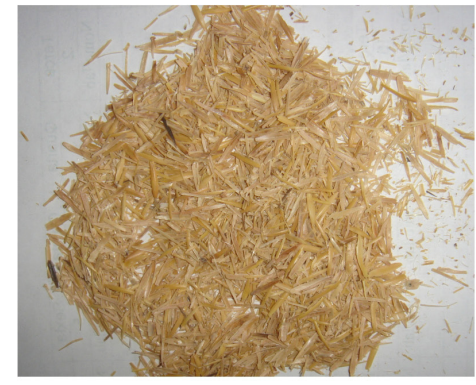

(a)

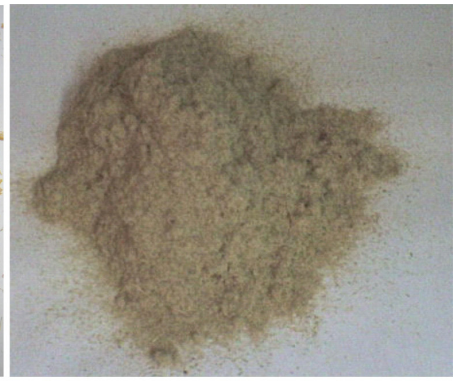

(b)

Figura 1S. Amostras de casca de aveia utilizadas no preparo dos compósitos: (a) amostra bruta e (b) amostra peneirada com classificação granulométrica - mesh 35

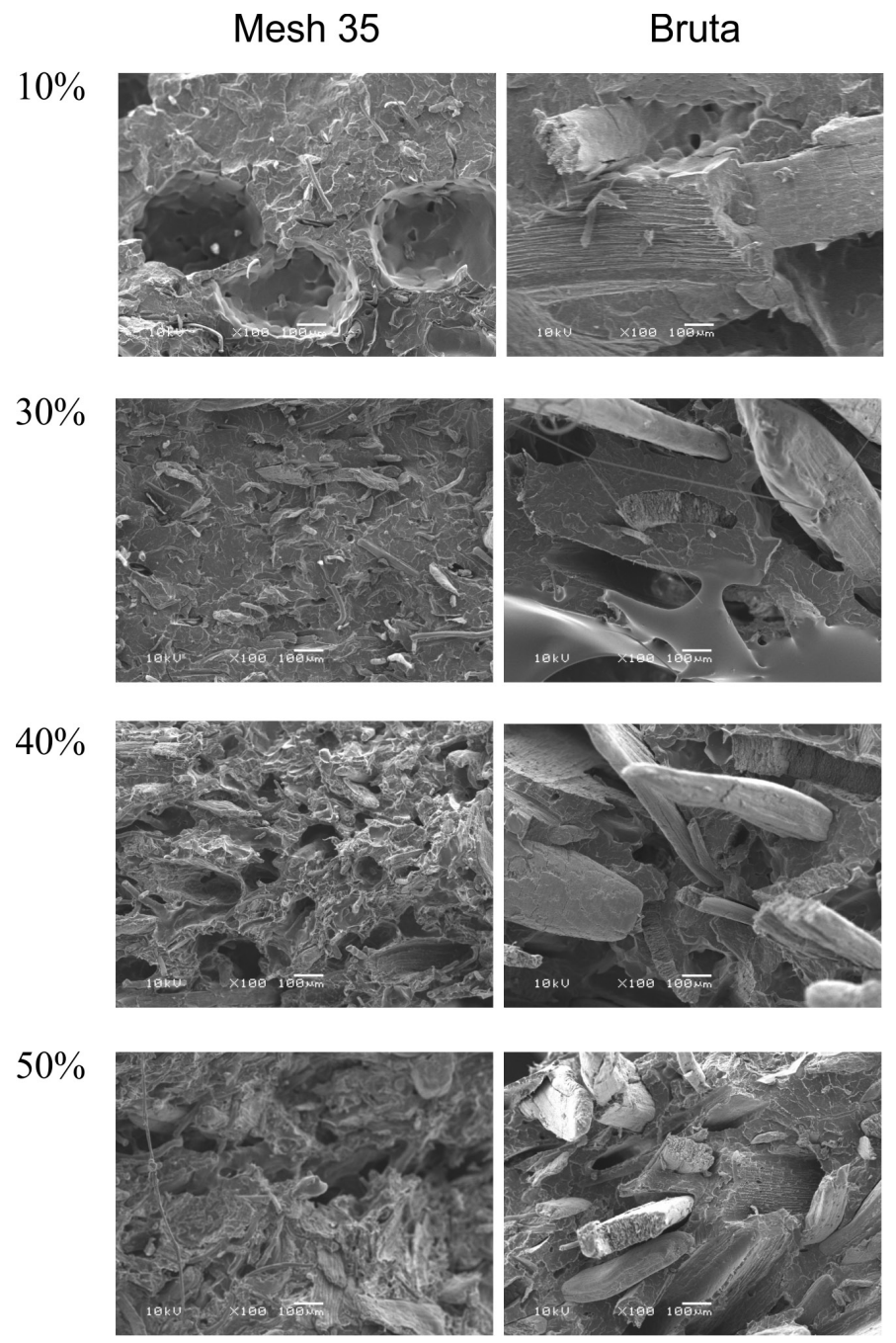

Figura 2S. Fotomicrografias MEV de superfícies criofraturadas dos compósitos de aveia (mesh 35) contendo diferentes teores de casca de aveia (bruta e mesh 35) 